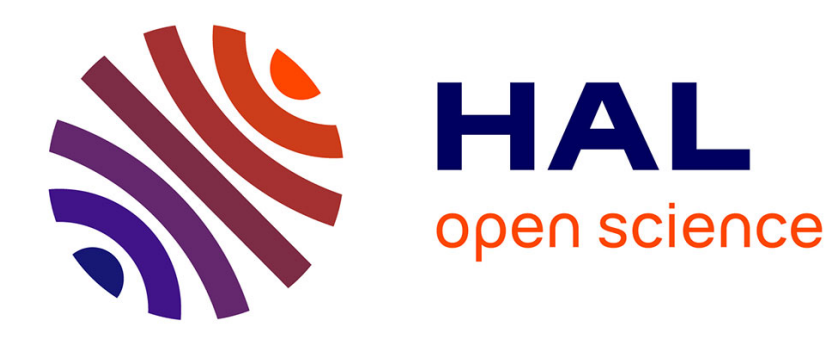

\title{
Weak and Strong Solutions of Dual Problems
}

\author{
Jean Jacques Moreau
}

\section{To cite this version:}

Jean Jacques Moreau. Weak and Strong Solutions of Dual Problems. Contributions to Nonlinear Functional Analysis, Apr 1971, Madison, WI, United States. pp.181-214. hal-01864005

\section{HAL Id: hal-01864005 https://hal.science/hal-01864005}

Submitted on 30 Aug 2018

HAL is a multi-disciplinary open access archive for the deposit and dissemination of scientific research documents, whether they are published or not. The documents may come from teaching and research institutions in France or abroad, or from public or private research centers.
L'archive ouverte pluridisciplinaire HAL, est destinée au dépôt et à la diffusion de documents scientifiques de niveau recherche, publiés ou non, émanant des établissements d'enseignement et de recherche français ou étrangers, des laboratoires publics ou privés. 


\section{Weak and Strong Solutions of Dual Problems}

\section{J. J. MOREAU}

§1. Introduction.

Many problems arising from various domains of physics consist in the investigation of functions defined, for instance, on a subset $S$ of $\mathbb{R}^{n}$, fulfilling certain requirements at each interior point of $S$ and other requirements at each boundary point. Such conditions, expressing physical laws of local character, generally involve continuity of the functions and their partial derivatives up to a certain order. At this stage of the problem, physicists frequently characterize the solution, if it exists, by variational properties or even extremal properties: it is that element of a certain class of functions where a certain functional attains its minimum.

These variational characterizations of solutions have suggested to mathematicians the idea of shifting from the socalled strong formulation of problems - i. e. the naive formulation provided by physics - to milder systems of requirements, yielding solutions denoted as weak and whose exist ence is more easily established. For instance, the function space to which the expected solution of the physical problem belongs is endowed with a topology (and a structure of uniform space); a minimizing sequence of the functional in question does not necessarily converge relatively to this topology, but in some typical cases one proves that convergence is achieved by imbedding the function space in its 
completion: the element of this completion to which the sequence converges is called a weak solution of the problem, and it is proved that it coincides with the so-called strong solution - i.e. the solution agreeing with the strong formulation- whenever the latter exists.

Another common feature in this connection is the duality between variational properties of solutions. In the classical case where the conventional machinery of the calculus of variations can be applied (this means that the considered functionals are, in some sense, differentiable on the function spaces where they are defined) this duality is well known: see Courant, Hilbert [1]. Dirichlet's problem gives the primary example of such a situation: Let $\Omega$ be a bounded open subset of $\mathbb{R}^{n}$; one looks for a numerical function $u$, continuous on the closure $\bar{\Omega}$, harmonic on $\Omega$ and coinciding with a prescribed continuous function on the boundary $\partial \Omega$. Let us suppose here that the boundary data can be extended as a function $\mathrm{f}$, continuous on $\bar{\Omega}$, possessing a square integrable gradient on $\Omega$. A first extremal characterization of the solution $u$ (if it exists) is that, in the set of the functions $\mathrm{v}$, continuous on $\bar{\Omega}$, agreeing with $\mathrm{f}$ on $\partial \Omega$, possessing a square-integrable gradient on $\Omega$, this solution is the (unique) element that realizes the minimum of the functional

$$
\mathrm{v} \rightarrow \int_{\Omega}|\operatorname{grad} \mathrm{v}|^{2} \mathrm{~d} \tau
$$

( $d \tau$ : volume or Lebesgue measure on $\Omega$ ). As a dual characterization of $u$, or rather of the vector field grad $u$, we have the following: in the set of the square-integrable vector fields on $\Omega$ which are the gradients of harmonic functions, grad $u$ is the (unique) element for which the functional

$$
w \rightarrow \int_{\Omega}|w-g r a d f|^{2} d \tau
$$

achieves its minimum (for more sophisticated versions of socalled Dirichlet's principle see e.g.: Brelot [1], [2]; Deny, Lions [1]). The underlying geometric pattern is that of projecting a known point of a certain pre-Hilbert space onto two 
orthogonal affine manifolds. These two projections are known to be complementary to each other by their common equivalence to a third operation of a different sort, the decomposition of a given element of the pre-Hilbert space into the sum of two elements belonging respectively to a certain pair of orthogonal vector subspaces.

In what follows we present a rather general model involving such triplets of equivalent ways of characterizing elements in a function space which is a priori not complete: two minimization properties, said to be dual to each other, and a decomposition property. This is done for nonlinear problems, without differentiability being considered for the functionals in question, but under certain convexity hypotheses. It is stressed that when imbedding the functional space initially considered into its completion, each of the three properties induces, in a natural way, a concept of weak solution, but these extensions do not in general agree with each other; in other words, the weakening procedure may not preserve duality. The core of this report consists in formulating a series of necessary and sufficient conditions for the consistency of the weakening procedures.

For sake of clarity we restrict ourselves to the case where the function space under consideration is endowed with a pre-Hilbert structure, as in the above example of Dirichlet's problem. Such a space making with itself a dual pair, the elements involved in dual minimization properties all belong to the same space, allowing a simple form for the associated decomposition pattern. Such a pre-Hilbert structure is usual in physics: the quadratic form whose polar form provides the scalar product will represent a kinetic energy, an electromagnetic energy, the potential energy of a linear elasticity law, the dissipation function of a linear viscosity law, etc...

First, in Section 2, we recall the necessary basic elements of the duality theory of convex functions defined on vector spaces. This theory, which originated in an idea of W. Fenchel [1], [2] has been extensively developed in recent years as a systematic tool for bringing out duality in convex extremal problems, without differentiability assumptions. 
For detailed accounts and bibliography the reader might refer to R. T. Rockafellar's book [3], devoted to finite-dimensional spaces but containing many guiding ideas for the general case, or, for a systematic study of convex functions defined on topological vector spaces, to J. J. Moreau [8]. More recent developments appear in J. L. Joly [1], M. Valadier [1], [2]. For applications to optimization problems in general or to the the ory of a pproximation see e. g. Rockafellar [2], Laurent-Joly [1], Moreau [12]. Following Rockafellar [1] and Ioffe-Tihomirov [1], the theory has been applied to functionals defined by integrals, thus to the calculus of variations and optimal control: see Rockafellar's contribution to this volume. In the same connection these methods have recently proved useful in the study of certain partial differential equa tions: see Temam [1], [2], [3], [4]. Subdifferential mappings, as defined in paragraph 2. $d$, provided the primitive example of monotone mappings- (G. J. Minty [1]).

The author's interest in this theory was motivated by the mechanics of continua (see: Moreau [4], [6], [10], [14] and Nayroles [1]).

From Section 3 onward, we restrict ourselves to preHilbert or Hilbert spaces. Proximation mappings-defined in this context generalize projections on convex sets; thus the present paper is connected to E. H. Zarantonello's article in this volume.

\section{$\S 2$. Elements of the Duality of Convex Functions.}

2.a. Polar functions.

Let $\mathrm{X}$ and $\mathrm{Y}$ be a dual pair of a real vector spaces; we denote by $\langle.,$.$\rangle the bilinear form which "puts these two$ spaces in duality". For instance $X$ may be a given topological vector space and $Y$ its topological dual, but in what follows $X$ and $Y$ will play perfectly symmetric roles. Each of the two spaces may be endowed with various topologies (actually equivalent when the dimension is finite) said to be compatible with the duality: on the space $X$, for instance, the se are the locally convex topologies relative to which the 
continuous linear forms consist exactly of the functions expressed as $\mathrm{x} \rightarrow\langle\mathrm{x}, \mathrm{y}\rangle$ with $\mathrm{y} \in \mathrm{Y}$ (and each continuous linear form on $\mathrm{X}$ corresponds to only one $\mathrm{Y} \in \mathrm{Y}$ if the usual separation condition is fulfilled). Among these topologies, the Mackey topology $T(X, Y)$ is the finest, the weak topology $\sigma(X, Y)$ is the coarsest.

An affine function on $\mathrm{X}$ (one should understand: continuous for topologies compatible with the duality) is written as

$(2.1)$

$$
x \rightarrow\langle x, y\rangle=\rho
$$

with $y \in Y$ (called the slope of the function) and $\rho \in \mathbb{R}$.

Let $f \in \overline{\mathbb{R}} X$ (i. e. a function defined on $X$ with values in $\overline{\mathbb{R}}=[-\infty,+\infty])$. The affine function (2.1) is, all over $\mathrm{X}$, a minorant of $\mathrm{f}$ if and only if
(2. 2)

$$
\rho \geq \sup \{\langle x, y\rangle-f(x): x \in X\}=f^{* *}(y)
$$

The function $\mathrm{f}^{*}$ defined thereby on $\mathrm{Y}$ is called the polar function of $f$ (or conjugate; in Rockafellar's book [3] the word "polar function" is used in another sense).

Example: Take as $f$ the indicator function $\psi^{\prime} s$ of a subset $S$ of $X$, i.e.

$$
\psi_{S}(x)=\left\{\begin{array}{cll}
0 & \text { if } & x \in S \\
+\infty & \text { if } & x \notin S
\end{array}\right.
$$

The polar function

$$
\begin{aligned}
\psi_{S}^{*}(y) & =\sup \left\{\langle x, y\rangle-\psi_{S}(x): x \in X\right\} \\
& =\sup \{\langle x, y\rangle: x \in S\}
\end{aligned}
$$

is conventionally called the support function of the set $\mathrm{S}$, with respect to the duality $(\bar{X}, \mathrm{Y})$. 


\section{2. b. Closed proper convex functions.}

Using the asterisk indifferently to denote the polarity from $\overline{\mathbb{R}}^{\mathrm{X}}$ to $\overline{\mathbb{R}}^{\mathrm{Y}}$ or from $\overline{\mathbb{R}}^{\mathrm{Y}}$ to $\overline{\mathbb{R}}^{\mathrm{X}}$, the polar $\mathrm{f}^{* * *}$ of $\mathrm{f}^{*}$ is

$$
\mathrm{f}^{* * *}(\mathrm{x})=\sup \left\{\langle\mathrm{x}, \mathrm{y}\rangle-\mathrm{f}^{* *}(\mathrm{y}): \mathrm{y} \in \mathrm{Y}\right\}
$$

From Condition (2.2) it is easily found that $\mathrm{f}^{* * *}$ equals the pointwise supremum of the affine functions such as (2.1) which minorize f .

Denote by $\Gamma(X, Y)$ the set of the numerical functions defined on $\mathrm{X}$ which are pointwise suprema of families of such affine functions. By standard separation arguments (i.e. the Hahn-Banach theorem), it may be proved that $\Gamma(\mathrm{X}, \mathrm{Y})$ consists of:

1. The constant function $+\infty$.

2. The constant function $-\infty$ (it is the supremum of an empty family of affine functions!)

3. The set of all functions called closed proper convex on $X$, namely the functions with values in

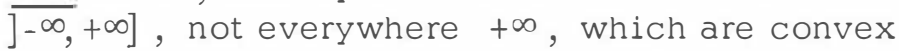
and lower semi-continuous for one (thus for all) of the (locally convex) topologies compatible with the duality $(\mathrm{X}, \mathrm{Y})$. This latter set will be denoted by $\Gamma_{0}(\mathrm{X}, \mathrm{Y})$.

Similar notations when exchanging $\mathrm{X}$ and $\mathrm{Y}$.

Example. The indicator $\psi_{S}$ of $S \subset X$ belongs to $\Gamma(X, Y)$ if and only if the set $S$ is convex and closed for one (thus for all) of the above topologies; $\psi_{S}$ belongs to $\Gamma_{0}(X, Y)$ if in addition $s$ is not empty.

Clearly $\mathrm{f}^{*} \in \Gamma(\mathrm{Y}, \mathrm{X})$ for any $\mathrm{f} \in \overline{\mathbb{R}}^{\mathrm{X}}$; furthermore $\mathrm{f}^{*} \in \Gamma_{0}(\mathrm{Y}, \mathrm{X})$ if and only if $\mathrm{f}$ is not everywhere $+\infty$ and possesses at least one affine minorant such as (2.1).

The function $\mathrm{f}^{* * *}$ is the greatest element of $\Gamma(\mathrm{X}, \mathrm{Y})$ minorizing $f$; thus it is called the $\Gamma$-hull of $f$.

In particular $\mathrm{f}=\mathrm{f}^{* * *}$ if and only if $\mathrm{f} \in \Gamma(\mathrm{X}, \mathrm{Y})$; this shows that polarity defines a one-to-one correspondence 
between $\Gamma(\mathrm{X}, \mathrm{Y})$ and $\Gamma(\mathrm{Y}, \mathrm{X})$. As the constants $+\infty$ and $-\infty$ are polar to each other, the correspondance is also oneto-one between $\Gamma_{0}(X, Y)$ and $\Gamma_{0}(Y, X)$.

In other words if it is supposed that $f \in \Gamma(X, Y)$ and $g \in \Gamma(Y, X)$, the relation $f=g^{*}$ is equivalent to $f^{*}=g$; in such a case $f$ and $g$ will be said to be mutually polar.

Example. Let $A$ and $B$ respectively be nonempty subset of $X$ and $Y$; it is easily found that the indicators $\psi_{A}$ and $\psi_{B}$ are mutually polar functions if and only if $A$ and $B$ are mutually polar cones, i. e. $A=\{x \in X:\langle x, y\rangle \leq 0$ for any $y \in B$ \} and symmetrically (such cones are convex, with vertex at the origin, and closed for the topologies compatible with the duality).

2. c. Lower semi-continuous hull and $\Gamma$-hull.

Suppose now that the function $\mathrm{f}$ is convex on $\mathrm{X}$, with values in $]-\infty,+\infty$ ], not everywhere $+\infty$; let $\underline{f}$ be its lower semi-continuous hull with regard to some (locally convex) topology compatible with the duality (X,Y), i.e. the greatest l.s.c. numerical function minorizing $f$. It is known that this l.s. c. hull may be constructed pointwise by

(2. 3) $\quad \underline{f}(x)=\lim _{u \rightarrow x} \inf _{x} f(u)$.

It is elementary that the convexity of $f$ implies the convexity of $\underline{f}$; thus, as soon as it is checked that $\underline{f}$ takes nowhere the value $-\infty$ (a common method for this is to check that $f$ possesses at least one continuous affine minorant) one concludes that $\underline{f} \in \Gamma_{0}(\mathrm{X}, \mathrm{Y})$ and, consequently, $\underline{\mathrm{f}}=\mathrm{f}^{* * *}$. , Observe at this stage that, due to the convexity of $f$ the choice of the topology when writing (2.3) is immaterial, as long as this (locally convex) topology is compatible with the duality $(\mathrm{X}, \mathrm{Y})$.

The following consequence of (2.3) will play an essential part in Section 4: Suppose that the restriction of f to its effective domain 


$$
\operatorname{dom} f=\{x \in X: f(x)<+\infty\}
$$

is lower semicontinuous (for the topology induced on this domain); then $f$ has the same restriction to this domain as f .

\section{2.d. Subgradients and subdifferentials.}

Let $\mathrm{f} \in \mathbb{R}^{X} ; \mathrm{Y} \in \mathrm{Y}$ is called a subgradient of $\mathrm{I}$ at the point $x$ if $y$ is the slope of an affine minorant of $f$ exact at the point $x$ (i.e. taking at this point the same value as $f$ ). This requires that the value $f(x)$ is finite and that the supposed minorant has the form

$$
u \rightarrow\langle u-x, y\rangle+f(x) .
$$

Using Condition (2.2) for an affine function to minorize $\mathrm{f}$, one obtains the following representation for the set, denoted by $\partial f(x)$, of the subgradients of $f$ at the point $x$

$$
\partial f(x)=\left\{y \in Y: f^{*}(y)-\langle x, y\rangle \leq-f(x)\right\}
$$

This set is called the subdifferential of $f$ at the point $x$. Clearly the convexity and the lower semicontinuity of $\mathrm{f}^{*}$ imply that $\partial f(x)$ is a convex (possibly empty) subset of $Y$, closed for the topologies compatible with the duality (Y,X). If $\partial f(x)$ is not empty the function $f$ is said to be subdiffer entiable at the point $x$.

Suppose for instance that the function $f$ is convex and weakly differentiable at the point $x$, with respect to the duality $(\mathrm{X}, \mathrm{Y})$ (such is a fortiori the case when $\mathrm{X}$ is a normed space, $Y$ itstopological dual with $f$ Fréchetdifferentiable at the point $x$ ) with the element $y \in Y$ as differential or "gradient"; then it is easily found that $\partial \mathrm{f}(\mathrm{x})$ consists of the single element $y$ (About relations between differentials and subdifferentials, see Asplund-Rockafellar [1]).

An evident use of the concept of subdifferential is the characterization of a minimum: the function $f$ attains 
its infimum at the point $x$ if and only if the set $\partial f(x)$ contains the origin of $Y$. There thus arises a need for a "subdifferential calculus "; in addition to Rockafellar's book [3] (for general bibliography and finite dimensional cases) the reader might refer to Moreau [7], [8], Valadier [1], [2]. The simplest rules of this calculus concern addition : if $\mathrm{f}_{1}$ and $f_{2}$ are numerical functions on $\mathrm{X}$, one trivially has, for any $x \in X$

$$
\text { (2.4) } \quad \partial \mathrm{f}_{1}(\mathrm{x})+\partial \mathrm{f}_{2}(\mathrm{x}) \subset \partial\left(\mathrm{f}_{1}+\mathrm{f}_{2}\right)(\mathrm{x})
$$

and it is important to formulate sufficient conditions for this inclusion to be an equality of sets. We shall only make use in this paper of this simple one (see Moreau [11]): If $\mathrm{f}_{1}$ and $\mathrm{f}_{2}$ are convex, one of them weakly differentiable at the point $x$, then the inclusion (2.4) is an equality of sets.

2.e. Conjugate points.

The preceding facts find their clearest ${ }^{\text {setting when }}$ one starts with a pair of mutually polar functions $f \in \Gamma_{0}(X, Y)$, $g \in \Gamma_{0}(Y, X)$. Then, for $x \in X$ and $y \in Y$, the three following properties are equivalent:
(2. 5)
$y \in \partial f(x)$

$$
x \in \partial g(y)
$$

$$
f(x)+g(y)-\langle x, y\rangle=0
$$

(Note that, by the definition of polarity, the left member of this latter equality is essentially nonnegative, whichever are $x \in X$ and $y \in Y$ ). In such a case the points $x$ and $y$ are said to be conjugate relative to the pair of mutually polar functions (f, g).

For instance, if $A \subset X$ and $B \subset Y$ are mutually polar cones, Condition (2.7) for $\mathrm{x}$ and $\mathrm{y}$ to be conjugate relative to the pair of mutually polar functions $f=\psi_{A}$ and $g=\psi_{B}$ becomes:

$$
x \in A, y \in B,\langle x, y\rangle=0
$$


In the very special case where $A$ and $B$ are two vector subspaces of $X$ and $Y$, each being the orthogonal of the other with respect to the duality, this latter condition reduces to: $x \in A$ and $y \in B$.

As a useful example of the equivalence between (2.5) and (2.6) let us note the following: The (possibly empty) set of the points where the function $f$ attains its infimum equals $\partial g(0)$, the subdifferential of the function $g$ at the origin of $Y$.

\section{2.f. Infimal convolution.}

Let $\mathrm{f}_{1}$ and $\mathrm{f}_{2}$ be two elements of $\overline{\mathbb{R}}^{\mathrm{X}}$; we denote by $\mathrm{f}_{1} \nabla \mathrm{f}_{2}$ the element of $\overline{\mathbb{R}}^{\mathrm{X}}$ defined as the function

(2.8) $x \rightarrow\left(f_{1} \nabla f_{2}\right)(x)=\inf \left\{f_{1}(u)+f_{2}(x-u): u \in X\right\}$

(or equivalently inf $\left.\left\{f_{1}(x-v)+f_{2}(v): v \in X\right\}\right)$.

The possible values of $u$ such that the above sum takes the indeterminate form $\infty-\infty$ must be omitted when constructing the "inf". The operation $\nabla$, called infimal convolution, is a commutative and associative composition law in $\overline{\mathbb{R}} X$. If $\mathrm{f}_{1}$ and $\mathrm{f}_{2}$ are convex, so is $\mathrm{f}_{1} \nabla \mathrm{f}_{2}$ (at least in an extended sense, since it may take the value $-\infty$ at some points: for the technique of overcoming such difficulties see Moreau [13]).

Example 1. If $\mathrm{f}_{2}$ is the indicator function of a singleton $\{\mathrm{a}\}$, then $\mathrm{f}_{1} \nabla \mathrm{f}_{2}$ is a translate of $\mathrm{f}_{1}$, namely the function

$$
x \rightarrow f_{1}(x-a)
$$

Example 2. If $\mathrm{A}$ is a subset of $\mathrm{X}$ and $\|\cdot\|$ a norm on this vector space, then $\left(\psi_{\mathrm{A}} \nabla\|\cdot\|\right)(\mathrm{x})$ is the distance of the point $x$ to the set $A$.

Taking now polar functions, easy computation yields

$$
\left(\mathrm{f}_{1} \nabla \mathrm{f}_{2}\right)^{*}=\mathrm{f}_{1}^{*}+\mathrm{f}_{2}^{*} \text {. }
$$


This fc-mula provides an answer to the following question: observing that addition is a composition law in $\Gamma(Y, X)$ (at least when extended by the convention $\infty_{-} \infty=-\infty$ ) and that polarity maps $\Gamma(Y, X)$ one-to-one onto $\Gamma(X, Y)$, what direct interpretation can be given of the composition law obtained in $\Gamma(X, Y)$ as the image of addition? Suppose that $\mathrm{f}_{1}$ and $\mathrm{f}_{2}$ belong to $\Gamma(\mathrm{X}, \mathrm{Y})$ and that $\mathrm{g}_{1}$ and $\mathrm{g}_{2}$, in $\Gamma(Y, X)$, are their polar functions. Eyuation (2.9), now written as

$$
\left(\mathrm{f}_{1} \nabla \mathrm{f}_{2}\right)^{* *}=g_{1}+g_{2}
$$

implies

$$
\left(g_{1}+g_{2}\right)^{*}=\left(f_{1} \nabla f_{2}\right)^{* * *}
$$

Thus the desired operation is the $\Gamma$-hull of infimal convolution.

This gives a great practical importance to cases where $\mathrm{f}_{1} \nabla \mathrm{f}_{2}$ happens to belong to $\Gamma(\mathrm{X}, \mathrm{Y})$ so that the double asterisk may be omitted. As already mentioned, the convexity of $f_{1}$ and $f_{2}$ guarantees that of $f_{1} \nabla f_{2}$; in view of paragraph 2. c. it just remains to check whether $\mathrm{f}_{1} \nabla \mathrm{f}_{2}$ is lower semi-continuous (with nowhere the value $-\infty$ ). This lies beyond the scope of the present paper (see Moreau [1], [8]).

3. Dual Extremum Problems in a Pre-Hilbert Space.

3. a. Definitions

Let $\mathrm{H}$ be a pre-Hilbert opace over the real numbers, i. e. a real linear space on which a certain positive definite quadratric form has been defined once ard for all; the square root of this quadratic form provides the norm $\|\cdot\|$ on $\mathrm{H}$; its polar form gives the scalar product of any two elements $x$ and $y$ of $H$, denoted by $(x \mid y)$. This bilinear form puts $H$ in duality with itself; as a topology compatible with this duality, the weak topology $\sigma(\mathrm{H}, \mathrm{H})$ will be considered; but it must be stressed that the norm topology is not compatible 
with the duality $(\mathrm{H} \mathrm{H})$ if $\mathrm{H}$ is not norm-complete, i.e. if it is not a Hilbert space.

Denote by $Q$ the quadratic form

$$
Q(x)=\frac{1}{2}\|x\|^{2} \text {. }
$$

Easy computation yields that $Q$ is Fréchet-differentiable, and also weakly differentiable relative to the duality $(\mathrm{H}, \mathrm{H})$, with

$$
\operatorname{grad} Q(x)=x
$$

The function $Q$ is convex; it belongs to $\Gamma_{0}(H, H)$ and equals its polar function.

Given $z \in H$, we shall denote by $Q_{Z}$ the function $\mathrm{x} \rightarrow \mathrm{Q}(\mathrm{z}-\mathrm{x})$, so that

$$
\operatorname{grad} Q_{z}(x)=x-z
$$

Let $\mathrm{f}$ be a convex numerical function defined on $\mathrm{H}$,

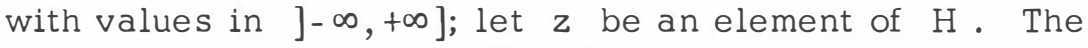
strict convexity of $Q$ implies that the function $f+Q_{Z}$ attains its infimum at at most one point (incidentally, this infimum is (f $\nabla \mathrm{Q})(\mathrm{z}))$; if such a point exists, it will be denoted by prox $(f ; z)$. The mapping $z \rightarrow \operatorname{prox}(f ; z)$ is called a proximation (Moreau [3]).

Example. Take as $\mathrm{f}$ the indicator function $\psi_{\mathrm{C}}$ of a nonempty convex subset $\mathrm{C}$ of $\mathrm{H}$; the point prox $\left(\psi^{\prime} \mathrm{C} ; \mathrm{z}\right)$, if it exists, is the nearest point to $\mathrm{z}$ in $\mathrm{C}$, frequently called the projection of $\mathrm{z}$ onto $\mathrm{C}$ and denoted by $\operatorname{proj}(\mathrm{C} ; \mathrm{z})$.

\section{3.b. Duality properties}

Proposition. Let $(\mathrm{f}, \mathrm{g})$ be a pair of mutually polar functions on $\mathrm{H}$ and let $\mathrm{z}$ be an element of $\mathrm{H}$.

If there exists prox $(f ; z)$, denoted by $x$, then there exists $\operatorname{prox}(g ; z)$, denoted by $y$; the points $x$ and $y$ are conjugate with respect to $(f, g)$ and $x+y=z$. 
Conversely if there exists a pair of points $x$ and $y$ which are conjugate with respect to (f,g) and such that $\mathrm{x}+\mathrm{y}=\mathrm{z}$, then $\operatorname{prox}(\mathrm{f} ; \mathrm{z})$ and $\operatorname{prox}(\mathrm{g} ; \mathrm{z})$ exist and equal $\mathrm{x}$ and $y$, respectively.

Proof: The relation $\mathrm{x}=\operatorname{prox}(\mathrm{f} ; \mathrm{z})$ means that the origin belongs to the subdifferential of the function $f+Q_{z}$ at the point $x$. By the differentiability of the function $Q_{z}$ and by the fact mentioned at the end of paragraph 2. $d$, this is equivalent to

$$
0 \in x-z+\partial f(x)
$$

or, when putting $y=z-x$, equivalent to $y \in \partial f(x)$, i. e. : the points $x$ and $y$ are conjugate with respect to $(f, g)$. From this equivalence, the proof is completed by exchanging $f$ with $g$ and $x$ with $y$.

Corollary 1. Let A and $B$ be a pair of mutually polar cones in $\mathrm{H}$ and let $z \in \mathrm{H}$.

If there exists $\operatorname{proj}(A ; z)$, denoted by $x$, then there exists $\operatorname{proj}(B ; z)$, denoted by $y$; one has $(x \mid y)=0$ and $\mathrm{x}+\mathrm{y}=\mathrm{z}$.

Conversely, if there exists a pair of points $x \in A$, $y \in B$, such that $(x \mid y)=0$ and $x+y=z$, then $\operatorname{proj}(A ; z)$ and $\operatorname{proj}(\mathrm{B} ; \mathrm{z})$ exist, respectively equal to $\mathrm{x}$ and $\mathrm{y}$.

Proof: Take $\mathrm{f}=\psi_{\mathrm{A}}$ and $\mathrm{f}=\psi_{\mathrm{B}}$ in above Proposition.

Particularizing more, we obtain:

Corollary 2. Let $\mathrm{F}$ and $\mathrm{G}$ be a pair of vector subspaces of $\mathrm{H}$, each being the orthogonal of the other, and let $z \in \mathrm{H}$. If there exists $\operatorname{proj}(F ; z)$, denoted by $x$, then there exists $\operatorname{proj}(G ; z)$, denoted by $y$, and $x+y=z$.

Conversely if there exists a pair of points $x \in F$ and $y \in G$ such that $x+y=z$, then $\operatorname{proj}(F ; z)$ and $\operatorname{proj}(G ; z)$ exist and equal $\mathrm{x}$ and $\mathrm{y}$, respectively. 
Proof: $\quad F$ and $G$ are mutually polar cones, like $A$ and $\mathrm{B}$ in the above Corollary; here $(\mathrm{x} \mid \mathrm{y})=0$ is automatically ensured by $x \in F$ and $y \in G$.

3. c. A functional example.

The following is a special case of a situation de scribed, in a context of hydrodynamics, in Moreau [4], [6]; such "unilateral" functional problems have been extensively studied in the recent years by various authors (see e.g. Brezis -Stampacchia [1 ], Lewy-Stampacchia [1], [2]).

Let $\Omega$ be a bounded open subset of $\mathbb{R}^{\mathrm{n}}$; take as $\mathrm{H}$ the space of the vector functions defined and continuous on the closure $\bar{\Omega}$ and which are the gradients of numerical functions. The scalar product of two elements $u$ and $v$ of $\mathrm{H}$ is defined by

$$
(u \mid v)=\int_{\Omega} u(\xi) \cdot v(\xi) d \tau(\xi)
$$

$(\xi$ : generic point of $\Omega ; \mathrm{d} \tau$ : Lebesgue measure; the dot represents the scalar product of the two elements $u(\xi)$ and $\mathrm{v}(\xi)$ of $\left.\mathbb{R}^{\mathrm{n}}\right)$

For the sake of simplicity we make in this paper the assumption that $\Omega$ is "very smooth", in order that:

i) there exists on $\partial \Omega$ a continuous field of outward normal unit vectors, denoted by $\xi \rightarrow v(\xi)$.

ii) The solutions of the conventional Dirichlet and Neumann problems exist, with gradient in $\mathrm{H}$ for any "very smooth" data on $\partial \Omega$ (say twice differentiable data, while $\partial \Omega$ is a twice differentiable hypersurface).

We are given:

1. An element $z=$ grad $r$ of $H$ (then the numerical function $r$ is finite and continuous on $\bar{\Omega}$ ).

2. A nonnegative "very smooth" function $b$ on $\partial \Omega$.

Call $\mathrm{C}$ the (convex) set of the elements $\mathrm{u}=\operatorname{grad} \mathrm{p}$ $\epsilon \mathrm{H}$ such that $\mathrm{p} \geq 0$ on $\Omega$ and $\mathrm{p}=\mathrm{b}$ on $\partial \Omega$. 
Call $D$ the (convex) set of the elements $v=$ grad $q$ $\epsilon \mathrm{H}$, such that $\operatorname{div} \vee($ i.e. the laplacian $\Delta q$ ), understood in the sense of distributions on the open set $\Omega$, is nonnega tive, i.e. is a nonnegative measure on $\Omega$; note that this measure is bounded, by virtue of the continuity of $\mathrm{v}$ on $\bar{\Omega}$.

Call $\mathrm{f}$ the indicator function of $\mathrm{C}$ and define another (convex, non finite) numerical function $\mathrm{g}$ on $\mathrm{H}$ as taking, for any $v=\operatorname{grad} q \in \mathrm{H}$, the following values ( $d \sigma$ denotes the area measure on $\partial \Omega$ ):

$$
\begin{array}{lrl}
g(v)=\int_{\partial \Omega} b v \cdot \operatorname{grad} q d \sigma, & \text { if } v \in D \\
g(v)=+\infty & , \quad \text { if } v \notin D .
\end{array}
$$

Decomposition problem. As it naturally arises from hydrodynamics this problem is formulated: to find in $\mathrm{H}$ the elements $\mathrm{x}=\operatorname{grad} \mathrm{p}$ and $\mathrm{y}=\operatorname{grad} \mathrm{q}$ such that

(3.1) $\quad x+y=z$

(3.2) $\quad x \in C, y \in D$

(3.3) $\quad p \Delta q=0$.

The latter condition means that the product of the nonnegative measure $\Delta \mathrm{q}$ and the continuous function $\mathrm{p}$ is the zero measure on $\Omega$; in view of (3.2) it may equivalently be written

$$
\int_{\Omega} \mathrm{p} \Delta \mathrm{q}=0 .
$$

Using an integration by parts, the joint conditions (3.2) and (3.3) are then found eyuivalent to

$$
f(x)+g(y)-(x \mid y)=0 .
$$

Thus the present situation will reduce to the pattern described in paragraph 3.b if we prove that $f$ and $g$ are mutually polar ( or in other words that the convex set $\mathrm{C}$ is 
closed for the topology $\sigma(\mathrm{H}, \mathrm{H})$ and that $\mathrm{g}$ is its support function). Actually the same integration by parts as above shows that the left member of (3.4) is non-negative for any $x$ and $y$ in $H$, i. e. each of the two functions $f$ and $g$ majorizes the polar of the other. Therefore we need only prove the two reverse inequalities:

1. Let us prove that $\mathrm{f} \leq \mathrm{g}^{*}$, i.e. for any $\mathrm{u}=\operatorname{grad} \mathrm{p}$ $\epsilon \mathrm{H}$

$$
f(u) \leq \sup \left\{(\operatorname{grad} p \mid \operatorname{grad} q)-\int_{\partial \Omega} b v \cdot \operatorname{grad} q d \sigma: \operatorname{grad} q \in D\right\}
$$

From $g$ being positively homogeneous it follows that $\mathrm{g}^{*}$ is an indicator. As $\mathrm{f}$ is the indicator of $\mathrm{C}$ and in view of the same integration by parts, this inequality reduces to the following statement: "if $u$ is such that

(3.5) $\sup \left\{-\int_{\Omega} \mathrm{p} \Delta \mathrm{q}-\int_{\partial \Omega}(\mathrm{b}-\mathrm{p}) v \cdot \operatorname{grad} \mathrm{q} d \sigma: \operatorname{grad} \mathrm{q} \in \mathrm{D}\right\}=0$ then $u \in C^{\prime \prime}$.

In fact, if $u$ fulfills condition (3.5), one may first choose $\mathrm{q}$ harmonic, and thus conclude that the integral

$$
\int_{\partial \Omega}(b-p) \nu \cdot \operatorname{grad} q d \sigma
$$

is nonnegative, and finally zero since $q$ may be changed into $-q$. Now $v$. grad q may be identified with any "very smooth" function having a zero integral over $\partial \Omega$ (this consists in solving a Neumann problem); thus $\mathrm{b}-\mathrm{p}$ is constant on $\partial \Omega$ or, adjusting the arbitrary constant in the determination of $\mathrm{p}, \mathrm{p}=\mathrm{b}$ on $\partial \Omega$. Therefore $(3.5)$ reduces to

$$
\int_{\Omega} \mathrm{p} \Delta \mathrm{q} \geq 0
$$

for any grad $y \in D$; now $\Delta q$ may be chosen with an arbitrary support in $\Omega$ and this yields the nonnegativity of $p$ all over $\Omega$.

2. Let us prove that $g \leq \mathrm{f}^{*}$, under the definition:

(3.6) $\mathrm{f}^{*}(\mathrm{v})=\sup \{(\operatorname{grad} p \mid \operatorname{grad} q): \operatorname{grad} p \in C\}$ 
where $\mathrm{v}=\operatorname{grad} \mathrm{q} \in \mathrm{H}$. First if $\mathrm{v}$ does not belong to $\mathrm{D}$ there exists an indefinitely differentiable nonnegative function $\varphi$, with compact support in $\Omega$, such that

$$
\langle\varphi, \Delta \mathrm{q}\rangle=-(\operatorname{grad} \varphi \mid \operatorname{grad} \mathrm{q})<0 .
$$

Then, by taking $\mathrm{p}=\mathrm{b}+\lambda \varphi$ ( $\mathrm{b}$ denotes here an extension of b to $\bar{\Omega}$, with gradient in $\mathrm{H}$ ) and giving to the positive number $\lambda$ arbitrarily large values, one proves that the right member of $(3.6)$ is $+\infty$.

Suppose now $v=$ grad $q \in D$; in view of the definition of $g$, we have to prove that, for any $\varepsilon>0$, there exists grad $p \in C$ such that

$$
\int_{\partial \Omega} \mathrm{b} \nu \cdot \operatorname{grad} q \mathrm{~d} \sigma \leq(\operatorname{grad} \mathrm{p} \mid \operatorname{grad} \mathrm{q})+\varepsilon
$$

or, using the integration by parts,

$$
\int_{\Omega} \mathrm{p} \Delta \mathrm{q} \leq \varepsilon .
$$

In fact, as $\Delta \mathrm{q}$ is a nonnegative bounded measure on $\Omega$, there exists $K$, a compact subset of $\Omega$, such that the integral of $\Delta q$ over $\Omega \backslash K$ is less than $\varepsilon / B$, where $B>0$ denotes a constant majorizing $\mathrm{b}$. As the function $\mathrm{p}$ may be chosen majorized by $\mathrm{B}$ and null over $\mathrm{K}$, this completes the proof.

3. d. Infimal convolution with $Q$.

We take again an arbitrary pre-Hilbert space and a pair (f, g) of mutually polar functions belonging to $\Gamma_{0}(\mathrm{H}, \mathrm{H})$. As $Q=Q^{*}$ and $f^{*}=g$, equation (2.9) yields

$$
(f \nabla Q)^{*}=g+Q \text {. }
$$

Since $g+Q$ is not everywhere $+\infty$, this proves that $f \nabla Q$ possesses affine minorants; thus this function takes nowhere the value $-\infty$; clearly also, by definition (2.8), it nowhere takes the value $+\infty$; the same conclusions apply to $g \nabla Q$. 
Now (3.7) implies

$$
(\mathrm{f} \nabla \mathrm{Q})^{* * *}=(\mathrm{g}+\mathrm{Q})^{*}
$$

where the right member may be computed as follows, for any $z \in \mathrm{H}$ :

$$
\begin{aligned}
(g+Q)^{*}(z) & =\sup \{(z \mid y)-g(y)-Q(y): y \in H\} \\
& =\sup \{Q(z)-g(y)-Q(z-y): y \in H\} \\
& =Q(z)-(g \nabla Q)(z) .
\end{aligned}
$$

As (f $\nabla Q$ ) $^{\text {*** }}$ minorizes $f \nabla Q$ (see Paragraph 2. b), this yields in particular the following inequality

$$
(\mathrm{f} \nabla \mathrm{Q})+(\mathrm{g} \nabla \mathrm{Q}) \geq \mathrm{Q} \text {. }
$$

The case of equality will be studied in Paragraph 5. a.

\section{Weakening Procedures and Duality.}

4.a. Strong problems.

Paragraph 3. b described a rather general situation involving a pair of extremum properties, formulated in the same pre-Hilbert space $H$, and said dual to each other: given $z \in H$, to find $x=\operatorname{prox}(f ; z)$ or to find $y=\operatorname{prox}(g ; z)$ appear as equivalent problems under the relation $\mathrm{y}=\mathbf{z}-\mathbf{x}$. Furthermore, this pair of equivalent minimization problems has been found equivalent to a third problem of a different sort: that of decomposing $z$ into the sum of two elements $x$ and $y$ which are conjugate relative to $(f, g)$. In what follows we shall refer to any of these three prcblems as a strong problem: such a problem does not necessarily possess a solution.

In the physical context, it is usually the decomposition problem which directly arises from phenomenological laws: the two minimization "principles" are subsequently 
derived. So, when going to the task of defining weak solutions of the physical problem variationally, no reason appears for preferring one of them to the other. Now the two choices do not in general provide the same concept of weak solution.

Remark 1. In order to obtain a simpler pattern of implica tions, we restrict ourselves in this paper to problems associated with a pair of mutually polar functions; actually several results may be obtained under the less stringent hypothesis that $f$ and $g$ are only "superpolar", i.e.: for any $x$ and $\mathrm{y}$ in $\mathrm{H}$ the inequality

$$
f(x)+g(y)-(x \mid y) \geq 0
$$

holds (this means that each of the two functions majorizes the polar of the other).

Remark 2. Results generalizing certain basic facts of the present theory may be formulated when considering, instead of pre-Hilbert or Hilbert spaces, some normed or Banach vector spaces (see Lescarret [1]).

4. b. The imbedding of $\mathrm{H}$ in its completion.

Let us denote by $\hat{\mathrm{H}}$ the Hilbert completion of the pre-Hilbert space $\mathrm{H}$; we shall treat $\mathrm{H}$ as imbedded in $\hat{\mathrm{H}}$, hence dense in it for the norm topology. $\hat{H}$ may as well be considered as the topological dual of $\mathrm{H}$ when this latter space is endowed with the norm topology. The scalar product in $\hat{\mathrm{H}}$ is an extension of the scalar product $(\cdot \mid \cdot)$ in $\mathrm{H}$ so that there is no need of a different notation for it. This bilinear form puts $\mathrm{H}$ in duality with itself; it puts in the same way $\hat{\mathrm{H}}$ in duality with itself; it also puts in duality the two spaces $\mathrm{H}$ and $\hat{\mathrm{H}}$. To the se three dualities correspond dis tinct classes of compatible topologies.

On $\hat{H}$, for instance one may consider:

(i) the norm topology, which is compatible with the duality $(\hat{\mathrm{H}}, \hat{\mathrm{H}})$ (this is actually the Mackey topology $\tau(\hat{H}, \hat{H}))$, 
(ii) the topology $\sigma(\hat{H}, \hat{H})$, coarser than the preceding one but compatible with the same duality, so that the family of the closed convex sets or the family of the l.s. c. convex numerical functions are the same for both, (iii) the topology $\sigma(\hat{\mathrm{H}}, \mathrm{H})$, coarser than $\sigma(\hat{\mathrm{H}}, \hat{\mathrm{H}})$.

On the subspace $\mathrm{H}$ of $\hat{\mathrm{H}}$, these three topologies respectively induce:

(i) the norm topology (this is actually the Mackey topology $\tau(\mathrm{H}, \hat{\mathrm{H}})$ ) compatible with the duality ( $\mathrm{H}, \hat{\mathrm{H}})$,

(ii) the topology $\sigma(\mathrm{H}, \hat{\mathrm{H}})$, coarser but compatible with the same duality,

(iii) the topology $\sigma(\mathrm{H}, \mathrm{H})$, coarser than $\sigma(\mathrm{H}, \hat{\mathrm{H}})$.

\section{4. c. Some extensions of $f$ and $g$ to $\hat{H}$.}

Let us start, as in Section 3, with a pair of numerical functions $\mathrm{f}$ and $\mathrm{g}$, belonging to $\Gamma_{0}(\mathrm{H}, \mathrm{H})$, mutually polar for the duality $(\mathrm{H}, \mathrm{H})$.

We shall denote by $f^{\prime}$ and $g^{\prime}$ their extensions to $\hat{\mathrm{H}}$ with the value $+\infty$ outside of $\mathrm{H}$; these extensions are convex on $\hat{\mathrm{H}}$.

The function $\bar{g}$ defined for every $y \in \hat{\mathrm{H}}$ by

$$
\begin{aligned}
\bar{g}(y) & =\sup \{(x \mid y)-f(x): x \in H\} \\
& =\sup \left\{(x \mid y)-f^{\prime}(x): x \in \hat{H}\right\}
\end{aligned}
$$

is indifferently the polar of $f$ for the duality $(H, \hat{H})$ or the polar of $\mathrm{f}^{\prime}$ for the duality $(\hat{\mathrm{H}}, \hat{\mathrm{H}})$; clearly it is an extension of $g$. Similarly $f$ is the polar of $g^{\prime}$ for the duality $(\hat{\mathrm{H}}, \mathrm{H})$; thus $\overline{\mathrm{g}}$ is the bipolar of $\mathrm{g}^{\prime}$ for the same duality, i.e., in view of paragraph 2. c, the 1.s.c. hull of $\mathrm{g}^{\prime}$ with respect to a topology on $\mathrm{H}$ compatible with the duality $(\hat{\mathrm{H}}, \mathrm{H})$, e.g. the topology $\sigma(\hat{\mathrm{H}}, \mathrm{H})$ (in fact $g^{\prime}$ possesses at least one continuous affine minorant since its polar function $f$ is not everywhere $+\infty$ ). 
The polar of $\bar{g}$ for the duality $(\hat{\mathrm{H}}, \hat{\mathrm{H}})$, i.e. the bipolar of $f^{\prime}$ for this duality will be denoted by $\hat{f}$; it is the l. s. c. hull of $\mathrm{f}^{\prime}$ relatively to any topology compatible with this duality, in particular the norm topology. As $f^{\prime}$ agrees with $f$ over the set (contained in $\mathrm{H}$ ) of the points where it differs from $+\infty$, and as $f$ is l.s. c. on $H$ for $\sigma(H, H)$ which is coarser than the norm topology, $\hat{f}$ is an extension of $f$ (see the end of paragraph 2. c.).

Symmetrically we define on $\hat{\mathrm{H}}$ the function $\hat{\mathrm{g}}$, namely the norm - l. s. c. hull of $\mathrm{g}^{\prime}$, and $\overline{\mathrm{f}}$, namely the $\sigma(\hat{\mathrm{H}}, \mathrm{H})-\mathrm{l}$. S. C. hull of $\mathrm{f}^{\prime}$, with similar polarity relations. The following inequalities hold:

$$
\begin{aligned}
& \overline{\mathrm{f}} \leq \hat{\mathrm{f}} \leq \mathrm{f}^{\prime} \\
& \overline{\mathrm{g}} \leq \hat{\mathrm{g}} \leq \mathrm{g}^{\prime} .
\end{aligned}
$$

4. d. Proximations in $\hat{\mathrm{H}}$.

The properties of proximations presented in Section 3 for an arbitrary pre-Hilbert space are true in the Hilbert space $H$ with this additional simplification: for arbitrary $\varphi \in \Gamma_{0}(\hat{\mathrm{H}}, \hat{\mathrm{H}})$ and arbitrary $\mathrm{z} \in \hat{\mathrm{H}}$, the point prox $(\varphi ; z)$ exists (uniquely). This is easily proved from the weak compactness of closed balls in a Hilbert space (see Moreau [3]) or by using general conditions for the "exactness" of infimal convolution (i.e. corditions for the infimum in (2.8) to be a minimum; see Moreau [1] [8]). $\hat{\mathrm{H}}$ is ensured

Therefore the existence of the following elements of

$$
\text { (4.1) } \hat{x}=\operatorname{prox}(\hat{f} ; z), \quad \bar{y}=\operatorname{prox}(\bar{g} ; z)
$$

these being equivalently characterized by

$$
\begin{gathered}
\hat{x}+\bar{y}=z \\
\hat{f}(\hat{x})+\bar{g}(\bar{y})-(\hat{x} \mid \bar{y})=0 .
\end{gathered}
$$


Symmetrically, the existence is ensured of
$(4.3)$
$\overline{\mathrm{x}}=\operatorname{prox}(\overline{\mathrm{f}} ;$
z),$\hat{y}=\operatorname{prox}(\hat{g}$;

equivalently characterized by

$$
\begin{gathered}
\bar{x}+\hat{y}=z \\
\bar{f}(\bar{x})+\hat{g}(\hat{y})-(\bar{x} \mid \hat{y})=0 .
\end{gathered}
$$

Proposition. Suppose $z \in \mathrm{H}$; if one of the four points $\overrightarrow{\mathrm{x}}, \overline{\mathrm{y}}, \overline{\mathrm{x}}, \hat{\mathrm{y}}$ happens to lie in $\mathrm{H}$, so do the other three and then

$$
\begin{aligned}
& \bar{x}=\hat{x}=\operatorname{prox}(f ; z) \\
& \bar{y}=\hat{y}=\operatorname{prox}(g ; z)
\end{aligned}
$$

(these proximations referring to $f$ and $g$ are evidently to be understood relative to $\mathrm{H}$ ).

Conversely, if $z \in \mathrm{H}$ and if there exists $\mathrm{x}=$ $\operatorname{prox}(f ; z)$ in $H$ (or equivalently by paragraph $3 . b$, if there exists $y=\operatorname{prox}(g ; z)$ ), equalities (4.4) and (4.5) hold.

Proof: This immediately follows from the above characterizations of "prox", since $f$ equals the restriction of $f$ or $\overline{\mathrm{f}}$ to $\mathrm{H}$ and $\mathrm{g}$ equals the restriction of $\hat{\mathrm{g}}$ or $\overline{\mathrm{g}}$ to $\mathrm{H}$. $\square$

At this stage, the replacement of $f$ and $g$, mutually polar numerical functions on $\mathrm{H}$, by their extensions $\hat{\mathrm{f}}$ and $\bar{g}$, mutually polar numerical functions on $\hat{\mathrm{H}}$, appears as a weakening procedure for the so-called strong problem of Paragraph 4.a: In fact this replacement yields a similar problem formulated in $\hat{\mathrm{H}}$ instead of $\mathrm{H}$ and which possesses a solution for any $\mathrm{z}$; this solution coincides with the solution of the strong problem whenever the latter exists.

The same is true for the replacement of $f$ and $g$ by their extensions $\bar{f}$ and $\hat{g}$, which yields another weakening procedure for the initial problem. More generally, one may choose $\tilde{\mathrm{I}} \in \Gamma_{0}(\hat{\mathrm{H}}, \hat{\mathrm{H}})$ such that 


$$
\overline{\mathrm{f}} \leq \tilde{\mathrm{f}} \leq \hat{\mathrm{f}}
$$

The polar function $\tilde{g}$ of $\tilde{\mathrm{f}}$ for the duality $(\hat{\mathrm{H}}, \hat{\mathrm{H}})$ satisfies

$$
\overline{\mathrm{g}} \leq \tilde{\mathrm{g}} \leq \hat{\mathrm{g}}
$$

and the same arguments as above show that, for a given $z \in H$, the elements $\operatorname{prox}(\tilde{\mathrm{f}} ; z)$ and $\operatorname{prox}(\tilde{\mathrm{g}} ; z)$ of $\hat{\mathrm{H}}$, respectively, coincide with $\operatorname{prox}(f ; z)$ and $\operatorname{prox}(g ; z)$ whenever one (then both) of the latter exist.

Thus we are in the presence of an infinity of weakening procedures for our initial "strong" problem.

4.e. Use of minimizing sequences.

Let $\varphi \in I_{0}(\hat{H}, \hat{H})$; a sequence $\left(u_{n}\right)$ of points of $\hat{H}$ is classically called a minimizing sequence of the function $\varphi+Q_{z}$ if

$$
\lim _{n \rightarrow+\infty}\left[\varphi\left(u_{n}\right)+Q_{z}\left(u_{n}\right)\right]=\inf \left\{\varphi(u)+Q_{z}(u): u \in \hat{H}\right\} .
$$

Standard arguments, essentially using the convexity of $\varphi$ and the uniform convexity of $Q_{Z}$, yield: Any minimizing sequence of $\varphi+Q_{z}$ converges to $\operatorname{prox}(\varphi ; z)$ relative to the norm topology of $\hat{\mathrm{A}}$.

Now we remark that, $\hat{f}$ being the norm-1.s. c. hull of $f^{\prime}$ and $Q_{z}$ being norm-continuous, the function $\hat{f}+Q_{Z}$ is the norm-l.s.c. hull of $f^{\prime}+Q_{z}$ and one has

$$
\inf \left\{f(u)+Q_{z}(u): u \in H\right\}=\inf \left\{\hat{f}(u)+Q_{z}(u): u \in \hat{H}\right\}
$$

(the same would not be true in general if we used instead of $\hat{\mathrm{f}}$ the function $\overrightarrow{\mathrm{f}}$, which is also an extension of $\mathrm{f}$ to $\hat{\mathrm{H}}$ but inferior to $\hat{\mathrm{f}})$. Therefore a sequence $\left(u_{n}\right)$ of points of $\mathrm{H}$ which in this space is minimizing for the function $f_{f}+Q_{Z}$ is also a minimizing sequence in $\hat{\mathrm{H}}$ for the function $\hat{\mathrm{f}}+\mathrm{Q}_{Z}$; thus: 
Proposition. Any minimizing seque्रnce of $f+Q_{z}$ in $H$ converges in the norm topology of $\mathrm{H}$ to the element $\operatorname{prox}(\hat{\mathrm{f}} ; \mathrm{z})$, denoted above by $\hat{\mathrm{x}}$; symmetrically any minimizing sequence of $g+Q_{z}$ in $H$ is normwise convergent in $\hat{H}$ to $\mathrm{y}=\operatorname{prox}(\hat{\mathrm{g} ; \mathrm{z})}$.

This proposition just describes the conventional weakening procedure for the two "strong" minimization problems in $\mathrm{H}$ whose the supposed solutions have been denoted by $\operatorname{prox}(f ; z)$ and $\operatorname{prox}(g ; z)$ (for a given $z \in \underset{\hat{H}}{H}$ ). Unfortunately the sum of the elements $\hat{x}$ and $\hat{y}$ of $\hat{H}$ does not in general equal $z$; in other words this weakening destroys the pattern of duality between the two minimization problems and also it destroys their equivalence with a decomposition problem.

Our purpose now is to formulate a series of necessary and sufficient conditions concerning $f$ and $g$, in order that the above minimizing sequence device preserves these basic features; at the same time these conditions will be necessary and sufficient for the various weakening procedures described in Paragraph 4. d to coincide.

\section{The Case of Uniqueness.}

5.a. Necessary and sufficient conditions.

Proposition. With the notations defined in the previous paragraphs, the following assertions are equivalent:

$$
\begin{aligned}
& \bar{f}=\hat{f} \\
& \bar{g}=\hat{g}
\end{aligned}
$$

(iii) Any pair $(\tilde{\mathrm{f}}, \tilde{\mathrm{g}})$ of mutually polar functions on $\hat{\mathrm{H}}$ which respectively minorize $f$ and $g$ on $H$ coincides with $(\hat{\mathrm{f}}, \hat{\mathrm{g}})$.

(iv) Any $z \in H$ equals the sum of $\hat{x}=\operatorname{prox}(\hat{f} ; z)$ and of $\hat{\mathrm{Y}}=\operatorname{prox}(\hat{\mathrm{g}} ; \mathrm{z})$ (let us recall here that $\hat{\mathrm{x}}$ and $\hat{\mathrm{y}}$ have been interpreted in Paragraph 4 . e as the limits in $\hat{\mathrm{H}}$ of minimizing sequences for the 
functions $f+Q_{z}$ and $g+Q_{z}$ in $H$ ).

(v) For any $z \in H$ and any $\varepsilon>0$ there exist $x_{\varepsilon}$ and $\mathrm{y}_{\varepsilon}$ in $\mathrm{H}$ such that

$$
\begin{array}{r}
\left\|x_{\varepsilon}+y_{\varepsilon}-z\right\| \leq \varepsilon \\
f\left(x_{\varepsilon}\right)+g\left(y_{\varepsilon}\right)-\left(x_{\varepsilon} \mid y_{\varepsilon}\right) \leq \varepsilon .
\end{array}
$$

(vi) Over all of $\mathrm{H}$ :

$(5.1)$

$$
\mathrm{f} \nabla \mathrm{Q}+\mathrm{g} \nabla \mathrm{Q}=\mathrm{Q} \text {. }
$$

(vii) The function $f \nabla Q$ (resp. $g \nabla Q$ ) is l.s. c. in $\mathrm{H}$ relative to the topology $\sigma(\mathrm{H}, \mathrm{H})$.

It must be stressed that properties (v) to (vii) only involve elements of the pre-Hilbert space $\mathrm{H}$ where the socalled strong problem was formulated; the same is true for property (iv) if it is interpreted in terms of minimizing sequences.

Property (v) was published in S. Maury [1]; it says that any $z \in \mathrm{H}$ can be "approximately decomposed" into the sum of two elements which are "approximately conjugate" relatively to (f,g) (in other words, $y_{\varepsilon}$ is an "approximate subgradient" of $\mathrm{f}$ at the point $\mathrm{x}_{\varepsilon}$; concerning this concept, see: Broendsted-Rockafellar [1]). It may equivalently be formulated as follows: for any $z \in \mathrm{H}$, there exists in the product space $\mathrm{H} \times \mathrm{H}$ a sequence $\left(\mathrm{x}_{\mathrm{n}}, \mathrm{y}_{\mathrm{n}}\right)$ which is minimizing at the same time for the two functions $(x, y) \rightarrow$ $\|x+y-z\|$ and $(x, y) \rightarrow f(x)+g(y)-(x \mid y)$ (in fact both functions are nonnegative and their infima are zero since $f$ and $\mathrm{g}$ are mutually polar).

Proof of the proposition: The equivalence between (i), (ii) and (iii) follows immediately from the fact that polarity reverses the ordering of functions.

Property (ii) implies the equality of the elements $\bar{y}$ and $\hat{y}$ as they are defined by (4.1) and (4.3); so property (iv) follows, by equality (4.2). Conversely, in view of (4.2), assertion (iv) implies that, for any $z \in \mathrm{H}$, 


$$
\operatorname{prox}(\hat{g} ; z)=\operatorname{prox}(\bar{g} ; z)
$$

But proximation mappings are norm-continuous from $\hat{\mathrm{H}}$ into itself (they are non-expanding: see Moreau [3]) and $\mathrm{H}$ is norm-dense in $\hat{\mathrm{H}}$. Therefore this equality also holds for any $z \in \hat{\mathrm{H}}$, implying that the functions $\hat{\mathrm{g}}$ and $\overline{\mathrm{g}}$ differ only by a constant (see Moreau [3]) ; this constant is zero since $\hat{g}$ and $\bar{g}$ agree with $g$ on $H$ (and $g$ is not everywhere $+\infty$ ). These assertions also imply $(v)$ : in fact consider in $\mathrm{H}$ a minimizing sequence $\left(\mathrm{x}_{n}\right)$ of the function $\mathrm{f}+\mathrm{Q}_{\mathrm{z}}$ and a minimizing sequence $\left(y_{n}\right)$ of the function $g+Q_{z}$. By paragraph 4.e these sequences are respectively normwise convergent in $\hat{H}$ to $\hat{x}$ and $\hat{y}$, whose sum equals $z$ according to assertion (iv); thereby $\left\|x_{n}+y_{n}-z\right\|$ is made arbi trarily small. Furthermore $\left(x_{n} \mid y_{n}\right)$ tends to $(\hat{x} \mid \hat{y})$ and, as the function $Q_{Z}$ is norm-continuous, $f\left(x_{n}\right)$ tends to $f(\hat{x})$ and $g\left(y_{n}\right)$ tends to $g(\hat{y})$ : then the second part of assertion $(v)$ follows from the fact that $f(\hat{x})+g(\hat{y})-(\hat{x} \mid \hat{y})$ is zero.

That conversely (v) implies (iv) may be deduced from the following identity:

$$
\begin{gathered}
f(x)+Q_{z}(x)+g(y)+Q_{z}(y)=Q(z)+Q(x+y-z)+f(x)+g(y) \\
-(x \mid y) .
\end{gathered}
$$

In fact property $(v)$ means that, for each $z \in H$, there exists a sequence $\left(x_{n}, y_{n}\right) \in H \times H$ which is minimizing for the right member of this identity, the infimum of which is $Q(z)$. Now

$$
\begin{aligned}
& \inf \left\{f(x)+Q_{z}(x): x \in H\right\}=(f \nabla Q)(z) \\
& \inf \left\{g(y)+Q_{3}(y): y \in H\right\}=(g \nabla Q)(z) .
\end{aligned}
$$

Comparing this with inequality (3.8), one finds that the sequence $\left(x_{n}\right)$ is minimizing for the function $f+Q_{z}$ and that the sequence $\left(y_{n}\right)$ is minimizing for the function $g+Q_{z}$; as the sequence $\left(x_{n}, y_{n}\right)$ is minimizing for the function 
$(x, y) \rightarrow Q(x+y-z)$, the sum $x_{n}+y_{n}$ is normwise convergent to $z$ and that implies (iv).

To finish the proof the same identity shows immediately that properties (v) and (vi) are equivalent. Furthermore paragraph 3.d yields

$$
(\mathrm{f} \nabla \mathrm{Q})^{* * *}+(\mathrm{g} \nabla \mathrm{Q})=\mathrm{Q}
$$

so that (5.1) is equivalent to

$$
(\mathrm{f} \nabla \mathrm{Q})^{* * *}=\mathrm{f} \nabla \mathrm{Q} \text {. }
$$

In view of paragraph 2. c this is just property (vii) .

The meaning of properties (vi) and (vii) becomes clearer in some special cases:

Special Case 1. Suppose that $f$ is the indicator function of a subset $\mathrm{C}$ of $\mathrm{H}$ (convex and $\sigma(\mathrm{H}, \mathrm{H})$-closed); for this particular form of $\mathrm{f}$, property (vii) means that the distance function

$$
z \rightarrow \operatorname{dist}(C, z)
$$

is $\sigma(\mathrm{H}, \mathrm{H})-\mathrm{l}$. S. C.

In fact $(f \nabla Q)(z)$ eyuals half the syuare of this distance.

Another equivalent assertion is: Any ball in $\mathrm{H}$, the radius of which is strictly smaller than the distance of its center to C, can be separated from $\mathrm{C}$ by a $\sigma(\mathrm{H}, \mathrm{H})$-closed hyperplane (i.e. a hyperplane possessing a normal vector in $\mathrm{H})$.

In fact the distance function is $\sigma(\mathrm{H}, \mathrm{H})-1$. s. C. if and only if, for any $\rho \geq 0$, the (convex) set $\{z \in H: \operatorname{dist}(\mathrm{C} ; \mathrm{z}) \leq \bar{\rho}\}$ is $\sigma(\mathrm{H}, \mathrm{H})$-closed. This in turn is equivalent to the separation property for any point $z_{0} \epsilon \mathrm{H}$ which does not belong to this set and, finally to the asserted separation property between $\mathrm{C}$ and a ball with center $\mathrm{z}_{0}$ and radius $\rho$. Actually direct arguments would easily show that this separation property between $\mathrm{C}$ and the balls in $\mathrm{H}$ 
is necessary and sufficient for the norm-closure of $C$ in $\hat{H}$ to coincide with the $\sigma(\hat{H}, H)$-closure of $C$ : this is just the form taken in the present case by property (i).

Special Case 2. Suppose that $f$ and $g$ are respectively the indicator functions of two mutually polar cones A and B. Then property (vi) reduces to the following "Pythagorean" relation

$$
[\operatorname{dist}(A ; z)]^{2}+[\operatorname{dist}(B ; z)]^{2}=\|z\|^{2}
$$

holding for any $z \in \mathrm{H}$.

5.b. Functional example.

Let us show that in the functional example presented in paragraph 3. C the preceding properties hold, in fact that assertion $(v)$ is verified.

The given continuous function $b$ on the (compact) set $\partial S 2$ was supposed nonnegative; just to simplify the following suppose here $b>0$.

Suppose $\mathrm{z}$ arbitrarily given in $\mathrm{H}$; thus $\mathrm{z}$ can be approximated as nearly as desired, in the sense of the norm, by an element grad $r \in \mathrm{H}$ such that $r$ is a "very smooth" numerical function on $\partial \Omega$ (see the smoothness assumptions made in paragraph 3. C).

First construct $y_{0}=$ grad $q_{0} \in D$ with the following
ies: properties:

$$
\begin{array}{ll}
\mathrm{q}_{0} \leq \mathrm{r} & \text { on } \bar{\Omega} \\
\mathrm{q}_{0}=\mathrm{r}-\mathrm{b} & \text { on } \quad \partial \Omega .
\end{array}
$$

For this purpose call $\gamma$ the Green potential of some negative smooth measure with compact support in $\Omega$ and call $\mathrm{h}$ the solution of the Dirichlet problem for the data $r-b$ on $2 S 2$. In view of our smoothness assumptions, the functions $\gamma$ and $\mathrm{h}$ have their gradients in $\mathrm{H}$; the function $\gamma$ is negative on $\Omega$; thus $\mathrm{q}_{0}=\mathrm{h}+\lambda \gamma$ fulfills above requirements when 
the positive constant $\lambda$ is chosen sufficiently large.

Our idea consists in applying Poincaré's sweeping out process to the function $\mathrm{q}_{0}$, but "conditionally" with regard to the inequality $\mathrm{q} \leq \mathrm{r}$.

Denote in general by $q$ a function satisfying the same conditions as above for $\mathrm{q}_{0}$, with gradient belonging to $\mathrm{D}$; the Laplacian $\Delta \mathrm{q}$ is a bounded nonnegative measure in $\Omega$. Let $B \subset \Omega$ be a closed ball; the function $q^{\prime}$ defined as equal to $q$ in $\bar{\Omega} \backslash B$ and equal in $B$ to the "harmonic interpolate" of q (i.e. the Poisson integral which solves the Dirichlet problem in the ball $B$ with $q$ as data on $\partial B$ ) is continuous on $\bar{\Omega}$, subharmonic on $\Omega$, greater than or equal to q. As q'agrees with q on a neighborhood of $\partial \Omega$, the nonnegative bounded measures $\Delta q$ and $\Delta q^{\prime}$ have the same total mass on $\Omega$.

Call "conditional harmonization of $q$ for $B$ under $r$ " the replacement of $q$ by $q^{\prime}$ if this latter function satisfies $q^{\prime} \leq r$, the conservation of $q$ if not.

Choose then a sequence of balls forming a base for the topology of $\Omega$; a classical construction yields another sequence in which each of these balls recurs infinitely. Starting with the function $\mathrm{q}_{0}$ constructed above, iterate conditional harmonization, using successively the balls of the latter sequence. This generates a non-decreasing sequence of continuous subharmonic minorants of $r$, all agreeing with $\mathrm{r}-\mathrm{b}$ on $\partial \Omega$; arguments in the style of the theory of potential (see Moreau [9]) prove that this sequence converges uniformly on each compact subset of $\Omega$ to a function $\bar{q}$ possessing the following properties: $\bar{q}$ is a continuous subharmonic minorant of $r$, agreeing with $r-b$ on $\partial \Omega$ and harmonic on the (open) subset of $\Omega$ where it differs from $r$ (in other words the nonnegative measure $(r-\bar{q}) \Delta \bar{q}$ is null). As we have supposed $b>0$ on $\partial \Omega$, the set $K$ on which $\bar{q}=r$ is a compact (possibly empty) subset of $\Omega$. Therefore, given $\varepsilon>0$, one can stop the process of successive harmonizations after a finite number of steps, obtaining a function $q_{1}$ which fulfills the following requirement: The difference $r-q_{l}$ is uniformly less than $\varepsilon$ on a compact set $K_{l}$, with $\mathrm{K} \subset \mathrm{K}_{\mathrm{l}} \subset \Omega$. This compact set can be constructed with smooth 
boundary; perform an ultimate "harmonization" by replacing $q_{1}$ on $\Omega \backslash K_{1}$ by its harmonic interpolate, without alteration on $\mathrm{K}_{1}$. The function $\mathrm{q}_{2}$ constructed thereby is again a continuous subharmonic minorant of $\bar{q}$ (since this latter is harmonic on $\Omega \backslash K_{1}$ ) thus of $r$; it agrees with $r-b$ on $\partial \Omega$. The nonnegative measure $\Delta q_{2}$ has a total mass on $\Omega$ not greater than the total mass $\mu$ of $\Delta q_{0}$ : in other words our initial measure $\Delta q_{0}$ has been "swept out" to the boundary of $\Omega$ and to a region where $r-q_{2} \leq \varepsilon$. This yields the inequality

$$
\int_{\Omega}\left(r-q_{2}\right) \Delta q_{2} \leq \mu \varepsilon .
$$

But $\mathrm{q}_{2}$ possesses a piecewise continuous gradient, square integrable on $\Omega$; by putting $r-q_{2}=p_{2}$, this allows an integration by parts transforming this inequality into

$$
\int_{\partial \Omega} p_{2} v \cdot \operatorname{grad} q_{2} d \sigma-\int_{\Omega} \operatorname{grad} p_{2} \cdot \operatorname{grad} q_{2} d \tau \leq \mu \varepsilon
$$

while

$$
\operatorname{grad} \mathrm{p}_{2}+\operatorname{grad} \mathrm{q}_{2}=\operatorname{grad} \mathrm{r} .
$$

It remains only to approximate, in the sense of the $\mathrm{L}^{2}$ norm, the vector fields grad $\mathrm{p}_{2}$ and $\operatorname{grad} \mathrm{q}_{2}$ by elements grad $p$ and grad $q$ of $H$, respectively belonging to the subsets $C$ and $D$ (alternatively one could have, in the previous process, "smoothed the transitions" after each step of harmonization, so as to deal all the time with functions having their gradients in $\mathrm{H}$ ); grad $\mathrm{r}$ was defined as approximating $z$. In that way one constructs elements $x=\operatorname{grad} p$ and $y=\operatorname{grad} q$ of $\mathrm{H}$, the sum of which is approximately $z$, and such that $f(x)+g(y)-(x \mid y)$ is approximately zero, with $f$ and $g$ defined as in paragraph 3.c. This is precisely property (v) of paragraph 5.a.

This example is obviously connected to the study of the regularity of solutions of certain variational inequalities, as developed in Lewy-Stampacchia [1], [2]. 


\section{REF ERENCES}

E. Asplund, R. T. Rockafellar [1] Gradients of convex functions, Trans. Amer. Math. Soc., 139 (1969), p. 443 467.

M. Brelot [1] Etude et extension du principe de Dirichlet, Ann. Inst. Fourier, 5 (1953-54), p. 371-419. [2] Eléments de la théorie classique du potentiel, Centre de Documentation Universitaire, Paris.

H. Brezis, G. Stampacchia [1] Sur la régularité de la solution d'inéquations elliptiques, Bull. Soc. Math. France, 96 (1968), p. 153-180.

A. Broendsted, R. T. Rockafellar [1] On the subdifferentiability of convex functions, Proc. Amer. Math. Soc., 16 (1965), p. 605-61l.

C. Castaing [1] Proximité et mesurabilité, Seminaire Anal. Unilatérale, Fac. Sci. Montpellier, vol. l (1968).

R. Courant, D. Hilbert [1] Methods of mathematical physics, Interscience publ., Vol. 1, Chap. 4, \$9 and 11.

J. Deny, J. L. Lions [1] Les espaces du type de Beppo Levi, Ann. Inst. Fourier, 5 (1953-54), p. 305-370.

W. Fenchel [1] On conjugate convex functions, Canad. Journ. Math. l (1949), p. 73-77.

[2] Convex cones, sets and functions, mimeographed lecture notes, Princeton University (1951).

A. D. Ioffe, V. M. Tihomirov [1] Duality of convex functions and extremal problems (Russian), Usp. Mat. Nauk

23 (1968), p. 51-116. 
J. L. Joly [1] Une famille de topologies et de convergences sur l'ensemble des fonctionnelles convexes, Thèse Sci. Grenoble (1970).

J. L. Joly, P. J. Laurent [1] Stability and duality in convex minimization problems, to appear.

C. Lescarret [1] Applications "prox" dans un espace de Banach, Compt. Rend. Acad. Sci. Paris, Ser. A, 265 (1967), p. 676-678.

H. Lewy, G. Stampacchia [1] On the regularity of the solution of a variational inequality, Comm. Pure Appl. Math., 22 (1969), p. 153 - .

[2] On the smoothness of superharmonics which solve a minimum problem, Journ. Anal. Math., 23 (1970), $227-236$.

J. L. Lions [1] Quelques methodes de résolution de problémes aux limites non linéaires, Dunod et Gauthier-Villars, Paris.

J. L. Lions, G. Stampacchia [1] Variational inequalities, Comm. Pure Appl. Math., 20 (1967), p. $493-519$.

S. Maury [1] Proximité dans un espace préhilbertien et complétion, Seminaire Anal. Convexe UER Math. Montpellier (1971).

G. J. Minty [1] On the monotonicity of the gradient of a convex function, Pacific J. Math. 14 (1964), 243-247.

J. J. Moreau [1] Inf-convolution des fonctions numériques sur un espace vectoriel, Compt. Rend. Acad. Sci. Paris, 256 (1963), p. $5047-5049$.

[2] Fonctionnelles sous-différentiables, Compt. Rend. Acad. Sci. Paris, 257 (1963), p. $4117-4119$. 
J. J. Moreau [3] Proximité et dualité dans un espace hilbertien, Bull. Soc. Math. France, 93 (1965), p. $273-299$.

[4] One-sided constraints in hydrodynamics, in: J. Abadie, editor, Nonlinear programming, North Holland Pub., Amsterdam (1967), p. $257-279$.

[5] Convexity and duality, in E. R. Caianiello, editor, Functional analysis and optimization, Academic Press, New York, 1966, p. 145-169. [6] Principes extrémaux pour le probléme de la naissance de la cavitation, Journ. de Mécanique, 5 (1966), p. $439-470$.

[7] Sous-différentiabilite, in Proceedings of the Colloquium on Convexity, Copenhagen 1965, p. 185 201.

[8] Fonctionnelles convexes, Séminaire sur les équations aux dérivées partielles, Collége de France, Paris 1967 (multigraph 108 p.)

[9] Majorantes sur-harmoniques minimales d'une fonction continue, Seminaire Anal. Unilaterale, Fac. Sci. Montpellier, vol. l (1968); an improved version is to appear in: Ann. Inst. Fourier 21, (1971). [10] La notion de sur-potentiel et les liaisons unilaterales en élastostatique, Compt. Rend. Acad. Sci. Paris, Ser A, 267 (1968), p. 954-957.

[11] Un cas d'addition des sous-différentiels, Séminaire Anal. Unilatérale, Fac. Sci. Montpellier, vol. 2 (1969).

[12] Distance a un convexe d'un espace normé et caractérisation des points proximaux, Séminaire Anal. Unilatérale, Fac. Sci. Montpellier, vol. 2 (1969). [13] Inf-convolution, sous-additivité, convexité des fonctions numériques, Journ. Math. Pures Appl. 49 (1970), p. $109-154$.

[14] Sur les lois de frottement, de plasticité et de viscosité, Compt. Rend. Acad. Sci. Paris, Ser. A, 271 (1970), p. 608-611. 
B. Nayroles [1] Essai de théorie fonctionnelle des structures rigides plastiques parfaites, Journ. de Mécanique, 9 (1970), p. 491-506.

R. T. Rockafellar [1] Integrals which are convex functionals, Pacific J. Math. 24 (1968), p. $867-873$.

[2] Duality in nonlinear programming, in Mathematics of the Decision Sciences, Part I, Lectures in Applied Mathematics, Vol II, Amer. Math. Soc. (1968), p. 401422.

[3] Convex Analysis, Princeton Univ. Press (1970). [4] This volume.

R. Temam [1] Remarques sur la dualité en calcul des variations, Compt. Rend. Acad. Sci. Paris, ser A, 270 (1970) p. $754-757$.

[2] Solutions généralisées de certains problémes de calcul des variations, Compt. Rend. Acad. Sci. Paris, ser. A, $271(1970$ p. $1116-1119$.

[3] Solutions généralisées de certaines équations du type hypersurfaces minima, to appear. [4] Solutions généralisées d'équations non lineaires non uniformement elliptiques, Pub. Math. Orsay, UER Math. Univ. Paris II, Centre d'Orsay (1970-71), 71 p.

M. Valadier [1] Sous-differentiels d'une borne supérieure et d'une somme continue de fonctions convexes, Compt. Rend. Acad. Sci. Paris, ser A, 268 (1969), p. $39-42$.

[2] Contribution à l'analyse convexe, Thèse Sci. Paris (1970).

E. H. Zarantonello [1]. This volume.

Faculte des Sciences Universite de Montpellier place Eugene Bataillon 34 Montpellier, France 